\title{
Hacia un enfoque social de los territorios inteligentes: una primera aproximación ${ }^{*}$
}

Towards a Social Approach to the Smart

Territories: A First Approach

$\grave{A}$ um enfoque social dos territórios inteligentes: uma primeira aproximação

Gloria Isabel Quintero Pérez ${ }^{* *}$

Recibido: 6 de diciembre de 2018

Aprobado: 11 de julio de 2019

Doi: https://www.doi.org/10.12804/revistas.urosario.edu.co/territorios/a.7487

Para citar este artículo:

Quintero Pérez, G. I. (2020). Hacia un enfoque social de los territorios inteligentes: una primera aproximación. Territorios, (42), 1-17. Doi: https://www.doi.org/10.12804/revistas.urosario.edu.co/territorios/a.7487

* Este artículo se consolida como resultado de la investigación de la autora en su cátedra: "Territorios Inteligentes. Ciudad-Región de Conocimiento", en el programa de Sociología de la Universidad de Antioquia $y$ el semillero de investigación con el mismo nombre, adscrito al grupo de Investigación Redes $y$ Actores Sociales, calificado como A por Colciencias.

* * Docente investigadora, Universidad de Antioquia. Correo electrónico: gisabel.quintero@udea.edu. co. ORCID: https://orcid. org/0000-0003-1391$017 X$ 
Palabras clave

Territorios, inteligencia, enfoque social, siglo XXI, territorio inteligente.

Keywords

Territories, intelligence, social approach, twentyfirst century, smart territory.

Palavras-chave

Territórios, inteligência, enfoque social, século XXI, território inteligente.

territarias 42

\section{RESUMEN}

Las aceleradas y disruptivas transformaciones que está viviendo la sociedad del siglo XXI han llevado al posicionamiento de nuevos modelos de comprensión y planeación de las ciudades y los territorios actuales. Modelos que integran la apuesta por la sostenibilidad ambiental, la incorporación de las Tecnologías de la Información y la Comunicación (TIC), la competitividad global y la cohesión y desarrollo social. Los territorios inteligentes son unos de los más abordados en nuestro contexto latinoamericano. ¿Qué son y de dónde surgen? ¿Dónde radica la verdadera inteligencia de un territorio? y ¿cuál es el enfoque social de los territorios inteligentes? Son las preguntas que orientan la construcción de este artículo, cuyo propósito es posicionar una mirada sociológica frente a este nuevo modelo de desarrollo territorial.

\section{ABSTRACT}

The accelerated and disruptive transformations that are taking place in the twenty-first century's society have led to the positioning of new models of understanding and planning of current cities and territories that integrate the commitment to environmental sustainability, the incorporation of Information and Communication Technologies, global competitiveness, and social cohesion and development. Smart territories are one of the most approached in the Latin American context. What are they and where do they come? Where is the real intelligence of a territory? What is the social focus of intelligent territories? These are the questions that guide the construction of this article, whose purpose is to position a sociological view in front of this new model of territorial development.

\section{RESUMO}

As aceleradas e disruptivas transformações que está vivendo a sociedade do século XXI têm levado ao posicionamento de novos modelos de compreensão e planeamento das cidades e os territórios atuais. Modelos que integram a aposta pela sustentabilidade ambiental, a incorporação das Tecnologias da Informação e a Comunicação, a competitividade global e a coesão e desenvolvimento social. Os territórios inteligentes, são um dos mais abordados em nosso contexto latino-americano. Que são e de onde surgem? Onde radica a verdadeira inteligência de um território? E que é o enfoque social dos Territórios Inteligentes? São as perguntas que orientam a construção deste artigo, cujo propósito é posicionar um olhar sociológico frente a este novo modelo de desenvolvimento territorial. 


\section{Introducción}

Es innegable que las sociedades del siglo XXI están viviendo la mayor transformación social, económica y urbana de la historia. Por primera vez, más del $50 \%$ de la población vive en ciudades y se espera que al 2050 sea más del $70 \%$; las ciudades se están posicionando como motores y protagonistas de la economía global; la tecnología digital ha significado una revolución a escala mayor, no solo en el sistema productivo mundial sino también en las formas de transmisión de la información y el conocimiento; este último, el conocimiento, se configura como el eje vertebrador de la nueva economía.

Tales cambios, y su vertiginosa velocidad, han implicado una incapacidad generalizada de respuesta desde las estructuras tradicionales, y una agudización de conflictos sociales, económicos y ambientales en los entornos urbanos de nuestra época. Por tanto, se fortalece la idea de que las ciudades, las regiones y los territorios deben transformarse y repensar asuntos básicos que les permita encontrar un equilibrio entre la cohesión y el desarrollo social, la competitividad económica y la sostenibilidad ambiental.

Para dar respuesta a estos asuntos y hacer uso del potencial de la tecnología digital en el siglo XXI, han surgido diferentes propuestas desde la última década del siglo XX: ciudades innovadoras, regiones que aprenden, ciudad-región, Smart cities, solo por mencionar algunas. Entre ellas, la idea de 'territorios inteligentes' se consolida como una tendencia creciente en el entorno de las políticas de sostenibilidad en las últimas dos décadas, desde que fuera acuñado el concepto en el 2004.

En la esencia del concepto subyace la inteligencia de los territorios, la capacidad de innovación de las personas, el apoyo de las tecnologías digitales para hacer más eficientes las ciudades e interconectar a los actores urbanos, de tal suerte que dicha inteligencia se vierta en la mejora de las condiciones y la calidad de vida de los territorios y sus habitantes. ¿ En dónde radica la verdadera inteligencia de los territorios? La respuesta está, indudablemente, en las personas, en la sociedad. No obstante, el foco de las discusiones sobre 'territorios inteligentes' solo ha tocado tangencialmente a las personas. ¿¿Cuál es entonces el enfoque social de los territorios inteligentes?

La primera parte de este artículo muestra la acelerada transformación que se está viviendo actualmente, bajo el título ¿época de cambios o cambio de época?; la segunda parte esboza algunos efectos sociales, económicos y ambientales problemáticos o inquietantes de dicha transformación; la tercera posiciona la idea de 'territorio inteligente' como una respuesta a estas transformaciones y problemáticas, donde se define el concepto y se describen sus principales características o atributos.

La última parte introduce la primera aproximación a un enfoque social de dichos territorios inteligentes a partir de territarias 42 
${ }^{1}$ La tendencia actual es a que cada vez se encuentran más personas, pero sobre todo más objetos conectados a la red. Según telefónica de España, entre 2010 y 2020 se pasará de tener 1.400 millones de objetos cotidianos conectados a tener 40.000 millones, a esta tendencia se le denomina internet de las cosas (IoT, por sus siglas en inglés). Adicionalmente, la digitalización de la economía lleva inevitablemente a cambiar la forma de vender: ya no se vende el producto sino el servicio (Roca, 2012).

\section{territarias 42}

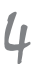

la pregunta ¿dónde radica la verdadera inteligencia de los territorios? Y esboza las primeras pinceladas de lo que busca ser dicho enfoque, como una apuesta sociológica para aportarle al modelo de territorio inteligente.

\section{¿Época de cambios o cambio de época?}

Si en algo hay coincidencia, tanto en el mundo de las ideas como en el mundo de la vida cotidiana, es frente a la acelerada velocidad de las transformaciones que está viviendo la sociedad, la economía y las ciudades del siglo XXI; algunos aducen que es la más grande y vertiginosa transformación en la historia de la humanidad, tanto, como para plantear que no estamos ante una época de cambios, sino ante un cambio de época (Alcalde, 2014; Vegara, 2009; Vegara y de las Rivas, 2016; Castells, 2009; Méndez, 2013; Arboleda, 2018).

El arqueólogo Genis Roca (2000) afirma que el dinamizador de tal transformación es el hecho digital, puesto que modifica de manera radical las formas de transmisión de conocimiento, el sistema productivo y las maneras de organizar el espacio, el trabajo y la sociedad. De hecho, si se hace un recorrido por los últimos treinta años de la historia, desde que se popularizó internet con la creación del World Wide Web en 1990 por Tim Berners-Lee, muchas cosas han cambiado, consecuencia directa o colateral: las formas de interacción social, las transacciones económicas, los modos de habitar el espacio, las prioridades sociales, las preocupaciones en el relacionamiento con la naturaleza y la ruralidad, la participación ciudadana, las estrategias de gobierno y la planeación de la ciudades, entre otras.

Todas estas transformaciones son aceleradas por tres acontecimientos globales: la revolución digital, el posicionamiento de la sociedad-economía del conocimiento y la concentración económico-demográfica en las ciudades del mundo. Es la primera vez en la historia de la humanidad que una tecnología - las TIC - afecta simultáneamente el sistema productivo y la forma como se transmite la información y el conocimiento (Roca, 2012); es también la primera vez que la mente humana es un factor de producción en sí mismo (Boisier, 2001; Marrero, 2007) y es también la primera vez que más del $50 \%$ de la población mundial vive en ciudades (Carrión, 2014). Se plantea que esta es la cuarta revolución industrial, caracterizada por ser intensiva en conocimiento, desarrollo tecnológico, innovación y explotación de datos (Departamento Nacional de Planeación, DNP, 2017).

La penetración de las TIC en la sociedad y la economía, además de facilitar la expansión de la globalización, está transformando las estructuras del sistema productivo - todo se está digitalizando-y la forma tradicional de transmitir información. Su uso posibilita la interacción permanente entre empresas, personas y objetos $^{1}$ de maneras diferentes, desde 
cualquier lugar y en tiempo real; además de la producción y el acceso a una cantidad inimaginable de información.

En medio de estas nuevas formas de interacción social digital, subyace la idea de la 'sociedad del conocimiento', popularizada en 1995 por Taichi Sakaiya y concebida en torno a la idea de que el conocimiento en términos abstractos es un elemento clave para el crecimiento económico y el desarrollo social de los territorios (Marrero, 2007). Como lo plantea Boisier, es precisamente el conocimiento el que subyace a los cambios disruptivos de los diferentes paradigmas sociales, científicos, productivos, organizacionales o de otra índole, frente a los que se enfrenta el mundo hoy (2001). Es decir, el hecho digital dinamiza las transformaciones, pero es el conocimiento el que las propicia.

Adicionalmente, la sociedad mundial actual es fundamentalmente urbana y la tendencia lo ratifica. El año 2008 se registra como el periodo de la historia en el que por primera vez más del $50 \%$ de la población mundial vive en ciudades, se estima que al 2050 será más del $70 \%$ y predicen que para el año 2025 tendremos más de 30 megaciudades ( 8 millones de habitantes o más), y más de 500 ciudades (un millón de habitantes) (Vegara, 2009). Según datos del BID (2014), en América Latina el porcentaje de población urbana al 2010 es ya del $80 \%$.

Aunado a lo anterior, se observa un posicionamiento protagónico de las ciudades en la economía global, lo que empieza a dinamizar redes globales de ciudades en el marco de la competitividad, y es en esta competencia donde adquiere una alta importancia la interdependencia creciente de los territorios (Vegara, 2009; Méndez, 2013), lo que significa que las ciudades ya no pueden pensarse solas, sino articuladas con sus entornos regionales y con otras ciudades a escala global, surge entonces la ciudad-región global (Pineda, 2009) como estrategia de competitividad territorial en un escenario donde se gana o se pierde.

\section{Algunos efectos inquietantes en el cambio de época}

Este cambio de época se caracteriza también por la agudización de varios asuntos problemáticos, como resultado de la vertiginosa transformación social, económica y urbana que se está experimentando, y por la incapacidad generalizada de las estructuras sociales tradicionales para adaptarse a dichas transformaciones y para responder a los conflictos sociales, económicos y ambientales que se agudizan.

Uno de los primeros asuntos, expresado de la mano de Joseph Stiglitz (2017), es que el malestar que produce la globalización en los países en desarrollo se ha globalizado, es decir, hay un malestar generalizado frente a la 'fractura' que produce la globalización, incluso en los países desarrollados, "si no gestionamos la globalización de manera que beneficie 
a todos, se corre el riesgo de que las reacciones negativas - que provienen de los nuevos malestares en el norte y los viejos malestares en el sur- se intensifiquen" (Stiglitz, 2017).

Un segundo elemento problemático es la ampliación de la brecha digital que ha representado la penetración de las TIC en las poblaciones más vulnerables, tanto en acceso como en uso y apropiación. Esta brecha representa la separación que hay entre quienes pueden acceder y usar estas tecnologías fácilmente y quienes no, y las desventajas que dicha situación conlleva. Por ello, además de dotar a las escuelas de computadores y conexión a la red, condición necesaria más no suficiente, es fundamental fortalecer las capacidades ciudadanas para usar, apropiar y producir tecnologías (Finquelievich, 2004)

En el caso colombiano, por ejemplo, se tiene que el número de conexiones fijas y móviles en el año 2017 es del $80 \%$, es decir, un $20 \%$ de la población está sin acceso a internet. Se observa también una separación de 18 puntos en el porcentaje de penetración de las TIC entre la región más conectada (Bogotá, con $21.8 \%$ ) y la menos conectada (Vichada con $1.3 \%$ ); y la ratificación de la brecha donde las regiones más fuertes tienen la mayor penetración (Bogotá-Cundinamarca, Antioquia, Santanderes, Eje cafetero, Valle del Cauca y Atlántico) y las más desfavorecidas, la menor penetración (Vichada, Putumayo, Guajira, Arauca y Chocó) (MinTIC, 2017, territarias 42
Segundo informe trimestral).
Otro efecto problemático que se ha impulsado con este cambio de época, más inquietante aún que la brecha digital, es la emergencia de una brecha cognitiva, referida a la separación entre quienes tienen acceso a la generación de conocimiento y quienes no. Por ejemplo, Estados Unidos tiene la cuarta parte de científicos del mundo, mientras América Latina y el Caribe solo el $3.5 \%$; además de que las siete naciones más industrializadas concentran el $90 \%$ de quienes participan en actividades científicas y tecnológicas (Marrero, 2007).

En la Sociedad del Conocimiento y la nueva economía es, precisamente, el conocimiento, como motor de innovación, el principal generador de transformación social, económica, ambiental y urbanística, por lo que la brecha cognitiva, o la falta de recurso humano cualificado, sumada a la brecha digital, representa una desventaja que afecta directamente la capacidad de agencia de las personas y el libre desarrollo de sus libertades, atentando contra los postulados del enfoque de Desarrollo Humano y de Derechos Humanos (Sen \& Nussbaum, 2016) y que pone a los territorios en un lugar desigual para competir en el escenario mundial.

Por otro lado, la acelerada urbanización en el mundo está planteando desafíos en términos sociales, infraestructurales y ambientales de un calibre extraordinario. Como plantea Vegara (2009), uno de los grandes retos de la humanidad es organizar las ciudades del siglo XXI. En 
términos simples, las grandes concentraciones económico-demográficas han generado un agotamiento de los recursos naturales, insostenibilidad en los procesos y una paradoja frente a la concentración de la riqueza y la desigualdad social.

Las ciudades son las principales productoras de gases de efecto invernadero con impactos evidentes en el calentamiento global, se estima que consumen el $75 \%$ de la energía mundial y generan el $80 \%$ de los gases de efecto invernadero (ONU-Habitat). Al mismo tiempo, concentran la mayor actividad económica para la generación de riqueza y, paradójicamente, una gran cantidad de población urbana se encuentra en condiciones de pobreza. Por ejemplo, en la región latinoamericana entre $60 \%$ y $70 \%$ del PIB regional se produce en las ciudades; sin embargo, $2 / 3$ de su población vive en condiciones de pobreza bajo patrones de segregación residencial y desigualdad (Carrión, 2014).

No obstante, algunos urbanistas notables coinciden en que las ciudades representan también la oportunidad para corregir dichos problemas (Vegara, Castells, Alcalde, BID, Boisier, Méndez), al ser el epicentro de una nueva estrategia: la innovación como elemento de alto valor estratégico en la economía y la sociedad, que conjuga las fortalezas o potencialidades de las transformaciones mencionadas: conocimiento y tecnología con las apuestas por la equidad, la competitividad y la sostenibilidad.

\section{Algunas respuestas al cambio: hacia la idea de 'territorio inteligente'}

En este contexto empiezan a emerger dos ideas complementarias: la primera es que las ciudades, las regiones, los territorios deben transformarse y repensar asuntos básicos que les permitan encontrar un equilibrio entre la trilogía: cohesión y desarrollo social, competitividad económica y sostenibilidad ambiental; la segunda aboga por la necesidad apremiante de actualizar el código de creencias y valores (Alcalde, 2015) con el que se estudian las ciudades, porque ante la magnitud de las transformaciones descritas, este código está desfasado.

Diferentes propuestas comienzan a posicionarse o a reciclarse en el escenario mundial: regiones que aprenden (Boisier, 2001), Smart Cities (Bouskela), territorios inteligentes (Vegara, 2004), ciudades innovadoras (Méndez, 2013), supercities (Vegara, 2016), ciudades con alma (Alcalde, 2015), ciudades a escala humana (Gehl, 2014), Smart City bumana (Moreno, 2014), entre otras. El punto de intercesión se encuentra en la transformación que se da en la manera de comprender y actuar sobre y desde las ciudades; estas nuevas maneras se soportan fundamentalmente en la relación estratégica entre el conocimiento, la tecnología y la innovación, en el marco de la ineludible responsabilidad global de responder a 
${ }^{2}$ Esta responsabilidad global es explicitada en los 17 Objetivos de Desarrollo Sostenible (ODS), acordados en la cumbre de las Naciones Unidas como horizonte de actuación al 2030 de los paises asociados.

Se prefiere hablar de territorios antes que, de ciudades, porque el concepto de ciudad en sí mismo excluye aquello que no es urbano, mientras el concepto de territorio es amplio y permite integrar el simbolismo, sentido y significado del espacio social como un todo que parte de la relación indisoluble entre lo urbano y lo rural, o que para efecto del articulo se entenderá como lo regional. Autores como Rafael Echeverri (2015), consideran, de hecho, que el territorio se convierte bajo este nuevo paradigma, en la nueva unidad de gestión pública.

\section{territarias 42}

estrategias de sostenibilidad centradas en las personas y su bienestar. ${ }^{2}$

El modelo de territorio inteligente integra, en su acepción más pura, muchos de los elementos postulados en otras propuestas como asuntos indispensables para transformar, actualizar y adaptar los territorios $^{3}$, las instituciones y las personas a las transformaciones mundiales actuales. Vegara y Rivas (2016) hacen un extraordinario recorrido por la historia del urbanismo para señalar de qué manera se ha llegado a las nuevas inquietudes de la arquitectura y el urbanismo en el siglo XXI. En este recorrido, iniciado en los orígenes del urbanismo moderno, se señalan tres grandes apuestas urbanas: la ciudadregión, el ecourbanismo y las Smart Cities como precursoras de lo que ellos han denominado 'territorios inteligentes'.

La ciudad-región evidencia lo que algunos ya habían intuido varias décadas antes, la urgencia territorial de concebir a las ciudades como parte de un ecosistema mayor, su región circundante, y las relaciones de complementariedad que allí se generan para ser más sostenibles y competitivas. El ecourbanismo establece una fuerte mirada en el paisaje de las ciudades y su relación cercana y responsable con el medio natural en el que se asienta. Las Smart Cities como ciudades que usan la tecnología y las tendencias (almacenamiento en la nube, uso masivo de dispositivos móviles, Big Data e Inteligencia Artificial) para procurar información del entorno y los elementos urbanos en tiempo real y tomar así decisiones más inteligentes.

El movimiento Smart Cities ha cobrado gran fuerza a nivel mundial, se ha escrito bastante y se han hecho varios congresos al respecto; sin embargo, la crítica frente a la preponderancia de la tecnología como el elemento que garantiza la inteligencia de la ciudad y la pregunta por la ruralidad ausente en el planteamiento, aunado al hecho de que los propulsores de este movimiento son las grandes empresas tecnológicas, ha hecho que incluso las reflexiones académicas increpen la propuesta y planteen el tránsito hacia la idea de territorios inteligentes, como lo muestra el lanzamiento del cuarto congreso mundial de ciudades inteligentes De la Ciudad al Territorio Inteligente: una evolución necesaria, realizado el 23 de enero de 2018 en la ciudad de Madrid.

Consolidar una definición sobre territorios inteligentes, parte de la definición inicial propuesta por Vegara y Rivas en 2004-2009 que plantea que los territorios inteligentes son territorios que han sabido dotarse de un proyecto de futuro compartido por los diferentes actores urbanos y basados en su identidad, en su idiosincrasia y especialmente en sus singularidades y sus componentes de excelencia, "capaces de encontrar un equilibrio entre los aspectos de competitividad económica; cohesión y desarrollo social; y sostenibilidad ambiental y cultural" (2009, p. 47), al articularla con otros desarrollos posteriores y complementarios y con las reflexiones propias en 
el marco de las investigaciones realizadas en el Departamento de Sociología de la Universidad de Antioquia, se consolida el siguiente concepto en construcción permanente:

Un territorio inteligente es aquel espacio socialmente construido, independiente de su tamaño, con un control administrativo, que ha logrado trazar un proyecto sostenible y transparente de futuro basado en su identidad y en sus singularidades, el cual es común a todos sus habitantes. Para diseñar este proyecto de futuro, es necesario que se posicione a las personas como eje y centro del desarrollo, donde su inteligencia trasciende la esfera individual y se filtra al conjunto de la sociedad. Como tal, el territorio inteligente es transparente y abierto a la comunidad, es competitivo económicamente a partir de sus virtudes particulares, es eficiente en el préstamo de los servicios públicos, posee una infraestructura pública que se articula con las plataformas digitales y propende por procesos sostenibles ambientalmente. Su principal característica es la creación de conocimiento, el aprendizaje continuo, la promoción de la innovación y el uso de enfoques diversos e incluyentes que posibilitan la interacción, la negociación y el acuerdo entre los diferentes actores y sus respectivos intereses; todo ello, con el fin de producir un mejor lugar para vivir, trabajar y compartir.

Lo que propone el concepto de territorios inteligentes es una nueva interpretación de conceptos como desarrollo competitivo y desarrollo sostenible aplicados al territorio del siglo XXI, para centrarlo en las personas, en su relación con el entorno natural, en su integración al mercado global y en la calidad de vida procedente de todo ello. De lo dicho anteriormente se deriva la comprensión de que, en principio, el territorio inteligente es un modelo de actuación frente a cualquier territorio, que tenga la intención de promover su inteligencia colectiva, independiente del tamaño que tenga.

Existen varias propuestas que buscan estructurar el modelo desde sus dimensiones, relaciones y atributos o elementos clave. Al hacer un rastreo por varias de ellas se encuentran elementos comunes a todas, que permiten la construcción de una estructura general para entender el modelo y que, seguramente, será rediseñado a partir del continuo aprendizaje y las nuevas experiencias, como un atributo innato de la inteligencia territorial (figura 1). Las principales dimensiones del modelo de Territorio Inteligente son las personas, las instituciones, la infraestructura, el ambiente y la economía, todo ello transversalizado por la tecnología, como herramienta o instrumento que, en el contexto de los territorios inteligentes, facilita la interacción, optimiza los recursos y eleva la posibilidad de tomar decisiones con base en información precisa y actualizada, y con la mirada puesta en la calidad de vida de las personas, pues como bien lo dice Carlos Moreno, lo demás es adorno (2014). territarias 42

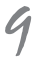


En relación con los elementos clave que caracterizan los territorios inteligentes, se pueden identificar siete básicos. En primer lugar, la apuesta por la sostenibilidad y la equidad, puesto de relieve en el informe de Desarrollo Humano de las Naciones Unidas del 2011, que plantea la dificultad natural de ocuparse de la protección del medio ambiente cuando la sobrevivencia es la prioridad y, por tanto, desde un punto de vista moral y práctico una mayor equidad, es un tema clave para alcanzar un desarrollo sostenible. En relación con este elemento, es necesario plantear que los territorios inteligentes deben incorporar enfoques que les permitan una gestión incluyente, una mirada especial y una adecuada priorización de recursos sobre los más vulnerables.

En segundo lugar, la capacidad continua de aprendizaje y adaptación, siendo el aprendizaje continuo un prerrequisito indispensable para una sociedad innovadora (Boisier, 2001; Méndez, 2002). A este elemento se le une un tercero, la incorporación de conocimiento, tanto interno como externo, la capacidad de conectarlo y utilizarlo en beneficio común, lo que en definitiva significa promover y aplicar la inteligencia colectiva.

Figura 1. Estructura de un modelo integrado de territorio inteligente

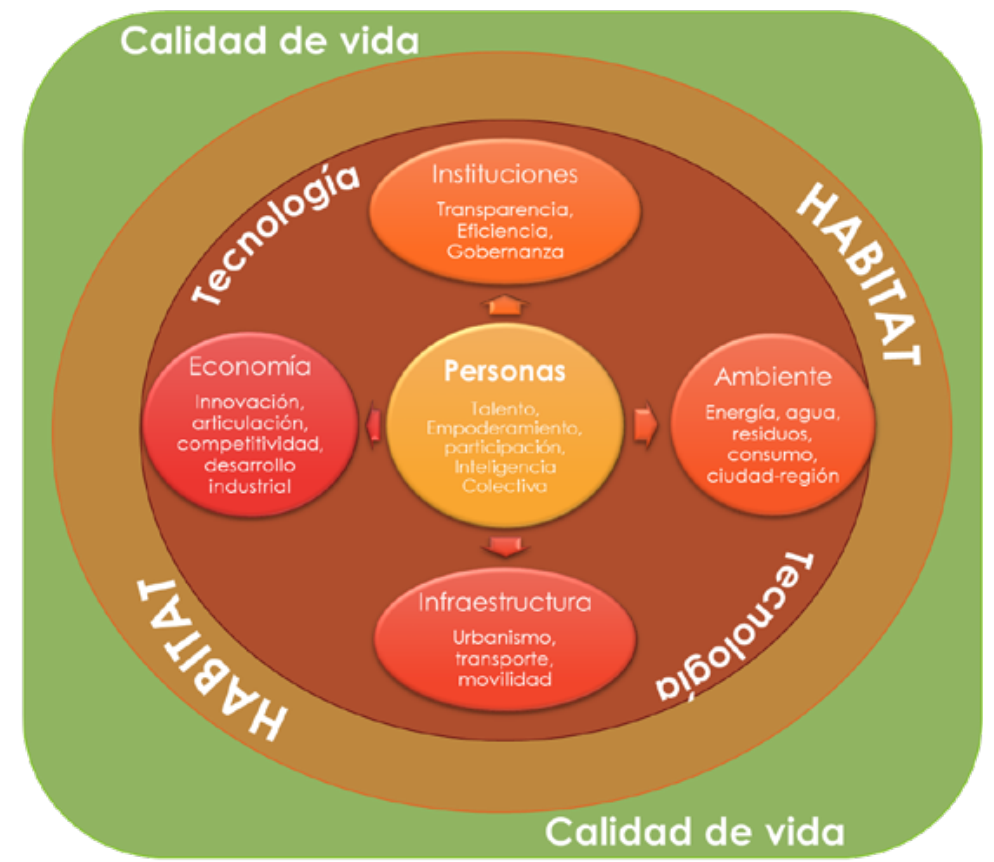

Fuente: elaboración propia. 
En cuarto lugar está el enfoque sistémico y la identificación de ventajas competitivas, que le permite al territorio reconocer su idiosincrasia y encontrar su singularidad competitiva en un marco global, a partir de la comprensión de sus relaciones sistémicas. Un quinto elemento es la gobernanza territorial, donde existe o se fortalece un liderazgo fuerte y una participación pública efectiva en la conformación de un proyecto de futuro basado en las expectativas y necesidades de las personas que viven en él, pues un territorio inteligente implica relaciones de colaboración entre los ciudadanos, el gobierno, las empresas y los centros educativos (Parada, 2017).

Lo que conduce a un sexto elemento clave: el trabajo en Red y la articulación con diferentes actores y con otros territorios que permitan ser más competitivos y potenciar la inteligencia de dicho territorio, pero, especialmente, trabajar por el desarrollo y la cohesión social. El séptimo atributo está relacionado con la innovación en todas sus esferas, especialmente la innovación social, como un elemento dinamizador del potencial territorial para afrontar problemáticas o situaciones particulares del territorio que requieren ser transformadas.

De acuerdo con la investigación desarrollada por la fundación Metrópoli en el proyecto CITIES que nació en 1998 con el objetivo de investigar 20 ciudades innovadoras de los cinco continentes, Vegara y Rivas (2016) en su libro La inteligencia del Territorio, supercities plantean algunos elementos comunes que caracterizan los territorios inteligentes y que contribuyen al entendimiento de los atributos y dimensiones del modelo propuesto. Los territorios inteligentes:

- Los diseña la comunidad.

- Asumen una nueva ética con respecto al medio ambiente natural y urbano.

- Son capaces de crear ventaja competitiva en relación con su singularidad.

- Son territorios en los que se trabaja para lograr la cohesión y el equilibrio social.

- Son espacios en los que existe una estructura coherente de gobierno del territorio.

- Encuentran las claves para el diálogo y articulación con su entorno.

- Son territorios que tienen un alto compromiso y le apuestan a la innovación.

- Participan de forma activa en redes de ciudades.

- Procuran una integración del mundo físico y virtual.

Podría entonces decirse que aquellos territorios con voluntad de construir su proyecto de futuro, basándolo en la sostenibilidad ambiental y cultural, en la inclusión social y tecnológica, en la responsabilidad empresarial, en el aprendizaje y la gestión del conocimiento, en la colaboración y la articulación y en la innovación, constituyen un territorio inteligente, para lo cual no se necesita un 
tamaño determinado, un nivel de infraestructuras dado o una fuerte penetración de la tecnología digital. Cualquier territorio puede ser un territorio inteligente (Calderero, et al., 2006).

\section{Hacia un enfoque social de los territorios inteligentes: ¿en dónde radica la verdadera inteligencia del territorio?}

En el discurso generalizado sobre territorio inteligente se ubica a las personas en el centro del modelo como principio y fin de toda la actuación. No obstante, las principales reflexiones y las apuestas en la implementación de territorios que se han embarcado en esta tarea: Masdar en Abu Dabi, Helsinki, Curitiba, Bilbao, Madrid, Copenhague, entre otros, señalan que este asunto es más discursivo que práctico. ¿Cómo lograr realmente que las personas estén en el centro, no solo como beneficiarios sino como protagonistas activos de la inteligencia del territorio?

La mayoría de las experiencias mundiales, que de una u otra forma están implementando el modelo de territorios inteligentes, han ubicado en el centro de sus actuaciones, generalmente, a la tecnología como el 'gadget' que trae consigo el germen de la inteligencia para una ciudad o territorio, y es precisamente este atributo el que se promueve con mayor ahínco. Y aunque la tecnología entrega información precisa en tiempo real sobre casi cualquier asunto que sea de interés, no es suficiente, porque, en últimas, siguen siendo las personas, en las instituciones, quienes toman las decisiones. Como lo dice Briales (2012), en el fondo no se trata de que las ciudades sean inteligentes, pues son un ente sin vida que no es inteligente en sí mismo, de lo que se trata es de que se pueda lograr que los ciudadanos en general puedan ser más inteligentes y tengan opción de serlo (Briales, 2012).

En el entorno científico y empresarial de esta nueva era, se puede respirar un ambiente de confianza exagerada en la tecnología y en la ciencia como la respuesta a las problemáticas que se enfrentan. Desde allí, es entendible que, por ejemplo, en Arabia Saudita se le entregara ciudadanía en 2017 a la primera robot mujer creada con Inteligencia Artificial: Sophia; o que empresas tecnológicas, como IBM, Cisco, INDRA, IDC, están revirtiendo sus negocios hacia las ciudades y proponiendo sus propios modelos de ciudades inteligentes.

Aunque la tecnología se constituye en un asunto clave para la inteligencia de un territorio, en últimas no es y no puede ser lo más importante, es un facilitador útil para que los ciudadanos puedan decidir cómo se mueven por la ciudad, cómo consumen, cómo se integran con otros ciudadanos; es importante en la medida que posibilita una mayor integración de los elementos de la ciudad y una mayor interacción entre los ciudadanos, las instituciones y los distintos sectores económicos (Conensa, 2012).

\section{territarias 42}


La verdadera inteligencia de un territorio no está en la mayor cantidad de artefactos tecnológicos que utilice, ni en la mayor integración al nuevo orden económico global, ni siquiera en aparecer en las listas y rankings internacionales. $\mathrm{La}$ verdadera inteligencia ha existido siempre en todos los pueblos y territorios, y está inextricablemente unida a sus tradiciones, sus saberes y su capacidad de respuesta a lo largo de su historia. Por ello, la verdadera inteligencia radica en la capacidad de promover el talento local al servicio de la calidad de vida de todos; está en el empoderamiento de la ciudadanía para hacerla partícipe directa del proyecto común de futuro; está en la capacidad de reconocer el potencial de sus gentes y su territorio, como Vegara y Alcalde lo llaman, en la capacidad de consolidar sus componente de excelencia desde donde pueda construir y potenciar alternativas innovadoras al servicio de todos, articulando tecnología, paisaje y personas.

Las ciudades y los territorios del siglo XXI necesitan, más que nunca, la innovación y la creatividad para diseñar su futuro. Las nuevas oportunidades que brindan las tecnologías digitales y las asombrosas nuevas posibilidades de interconexión y acceso a la información se aprovechan en todo su potencial cuando existe un Proyecto de Territorio que identifica iniciativas, establece prioridades y orienta las actuaciones públicas y privadas para lograr un perfil propio, apoyado en sus singularidades y en sus componentes de excelencia. Los territorios no solo son atractivos sobre la base de sus condiciones intrínsecas, sino que han sido capaces de consensuar un proyecto inteligente de futuro... Disponer de un Proyecto de Territorio es un elemento diferenciador que genera oportunidades y ventajas en los territorios que disponen de él. Es una forma viable de crear ventaja competitiva y magnetismo en la etapa de la globalización. Es, también, una tarea compleja que exige generar consensos y alianzas a largo plazo y aportar los recursos humanos, organizativos y económicos necesarios para convertir las ideas en realidad (Findeter, 2016, 14-15 y 26).

El enfoque social de los territorios inteligentes se encuentra en este lugar precisamente, en el reconocimiento fáctico de que la verdadera inteligencia está en las personas y en su capacidad para llegar a consensos territoriales sobre las potencialidades, las fortalezas y el proyecto de futuro colectivo al que todos, aún en medio de sus intereses particulares, le apostarán como una sola fuerza, en un solo sentir. Porque, como bien lo expresa Carlos Moreno, lo que cambia a la sociedad no es la tecnología, es la gente; precisamente la configuración de territorios inteligentes también depende de la agencia humana tanto individual como colectiva, de tal forma que desarrollen un peso específico de tipo institucional que empuje el proceso, si ello no existe, pueden existir formalmente los componentes para 
un territorio inteligente, $\mathrm{y}$, sin embargo, no es seguro que algo significativo suceda.

La verdadera inteligencia radica, como escribe Francisco Proenza (2002), citado por Finquelievich (2004) en que: "Los países que buscan prosperidad y estabilidad social [...] deberían focalizarse en la e-Para Todos, es decir, en hacer accesibles a todos los ciudadanos las oportunidades ofrecidas por las TICs para el desarrollo individual y social, y en usar las TICs para fortalecer a los ciudadanos comunes, para comprometerlos en iniciativas de desarrollo local y regional" (p. 11).

Complementando lo anterior, Carlos Moreno, precursor del movimiento ciudades vivas y Smart Cities humanas, plantea que la problemática central de las Smart Cities no es un asunto tecnológico, sino que en primer lugar es un asunto de gobernanza, donde la revolución tecnológica debe estar al servicio de la inclusión social y la inteligencia urbana, procurando que las personas salgan de su mundo tecnológico personal para convertirse en ciudadanos digitales (2015); y agrega que los desafíos de la inteligencia urbana son sociales, culturales, económicos, ecológicos y de resiliencia.

$\mathrm{Al}$ entender que un ciudadano es una persona que, en cooperación con otros, es capaz de crear y transformar el orden en que quiere vivir y proteger la dignidad de todos (Toro, 2001), - y que las redes sociales y el mundo digital han abierto una posibilidad inaprensible de interconexión

\section{tersitarias 42}

Ratti (2011) - una apuesta real de enfoque social de los territorios inteligentes busca la promoción del talento local, la formación de ciudadanos y el cierre de brechas cognitivas y digitales, en pro de ciudadanos digitales y ciudadanías emergentes, para revertir esta inteligencia sobre la calidad de vida de todos los habitantes del territorio.

De acuerdo con la conferencia de Rodrigo Arboleda en la cátedra de Innovación Empresarial de la Universidad Eafit el 4 de abril de 2018, la tecnología está creciendo de manera exponencial, lo que genera cambios radicales en los paradigmas establecidos, mientras que la estructura social se transforma de manera lineal, según su experiencia y conocimiento, son cuatro las tecnologías que van a cambiar el mundo: el vehículo autónomo eléctrico, la realidad virtual o aumentada, la nanotecnología y la inteligencia artificial con el análisis masivo de datos.

En este cruce, asevera que la sociedad no está lista para lo que se viene y que la apuesta que los territorios tengan por la educación y la transformación social va a ser la base fundamental para adaptarse a estos nuevos cambios. Adicionalmente, Salim Ismail en el video How do we fix Civilization (2016), aduce que es necesario crear nuevos tipos de organizaciones y un nuevo perfil de ciudadano, además de crear un nuevo contrato social. Argumentos que en última instancia remiten a corroborar que el verdadero enfoque social, la verdadera inteligencia de los 
territorios, está dada en poner la mirada sobre las personas y darles voz y poder, puesto que la vida surge cuando se ofrece a la gente la oportunidad de contribuir en algo y esto solo es posible cuando se brindan espacios para las personas (documental La escala humana, 2014).

Poner la mirada sobre las personas implica la inclusión de enfoques para el desarrollo en las actuaciones de las instituciones públicas y privadas que dirigen los asuntos del territorio inteligente, enfoques como: el Desarrollo Humano Sostenible, el basado en Derechos, el de Género, el de Reconocimiento e Inclusión Diferencial, el de Desarrollo Territorial y el de Seguridad Humana, entre otros, que permitan comprender la dinámica social de una manera particular y priorizar los recursos financieros y humanos para trabajar directamente y de manera decidida con las personas, en la consolidación del proyecto de futuro.

La verdadera inteligencia radica en que los territorios articulen esfuerzos con sus diferentes actores territoriales: ciudadanos, organizaciones, empresas, academias, instituciones para trabajar por la resolución verdadera y efectiva de las necesidades prácticas de su población y para mejorar la calidad de vida de todos, no desde la visión de unos privilegiados sino desde la visión colectiva y compartida del futuro deseado, en un ánimo de gobernanza territorial como la mayor expresión de inteligencia territorial.

\section{Referencias}

Alcalde, I. (2014). El futuro de las ciudades. Ponencia presentada en Tecnalia. Madrid, España. Recuperado de https://www.youtube.com/ watch? $=2$ hylMG615WI

Arboleda, R. (2018). Conferencia Cátedra de Innovación Empresarial. Medellín: Universidad Eafit.

Boisier, S. (2001). Sociedad del conocimiento, conocimiento social y gestión territorial. Celsa, 4, 61-94.

Bouskela, M. et al. (2016). La ruta hacia las smart cities. Migrando de una gestión tradicional a la ciudad inteligente. Washington D.C.: BID.

Briales, J. (2012). Smart cities en España. Recuperado de https://youtu.be/ nFRd7pTsmrQ

Calderero Gutiérrez, A. et al. (2006). Territorio inteligente y espacio de economía creativa: una primera aproximación conceptual y práctica de investigación. XVI Congreso de Estudios Vascos: Garapen Iraunkorra-IT, España, Donostia : Eusko Ikaskuntza.

Carrion, D. (2014). Liderando el Desarrollo Sostenible de las Ciudades. Unidad Temática Planificación urbana y ordenamiento territorial. Washington D.C. Banco Interamericano de Desarrollo (BID) y del Instituto Interamericano para el Desarrollo Económico y Social (INDES)

Castells, M. (2009). Comunicación y poder. Madrid: Alianza Editorial territarias 42

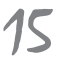


Colmenarejo, R. (2016). Enfoque de capacidades y sostenibilidad Aportaciones de Amartya Sen y Martha Nussbaum. Ideas y Valores, LXV (160), 121-149.

Colombia, Ministerio de Tecnologías de la Información y las Comunicaciones, MinTic. (2017). Informe trimestral. Segundo trimestre de 2017. Recuperado de www.mintic.gov.co/ colombiatic

Conensa, P. (2012). Smart cities en España. Recuperado de https://youtu.be/ nFRd7pTsmrQ

Departamento Nacional de Planeación, DNP. (2017). Planeando ciudades modernas. Foro económico mundial, DNP.

Findeter (2016). Libro blanco Diamante Caribe y Santanderes. Bogotá.

Finquelievich, S. (2004). La sociedad civil en la economía del conocimiento: TICs $y$ desarrollo socio-económico. Buenos Aires: Instituto de Investigaciones Gino Germani, Facultad de Ciencias Sociales, UBA

Gehl, J. (2014). Documental. La Escala Humana. Recuperado de https://www.youtube.com/

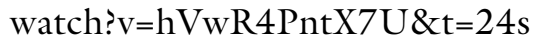

Marrero, A. (2007). La sociedad del conocimiento: Una revisión teórica de un modelo de desarrollo posible para América Latina. ARXIUs, 17, 63-73.

Méndez, R. (2013). Estrategias de innovación para el desarrollo y la resiliencia de ciudades medias. Documents d'Anàlisi Geogràfica, 59(3), 481-499.
Moreno, C. (2014). Smart CitiesHuman Cities. Conferencia TEDx PalmadeMallorca. Recuperado de https://www.youtube.com/ watch?v=AhXeka_Kd54

Parada, J. (2017). Innovaciones sociales para territorios "inteligentes": ¿ficción o realidad? Revista Problemas del Desarrollo, 190(48), 11-35.

Pineda-Hoyos, S. (2009). Ciudad-Región global: una perspectiva de la gobernanza democrática territorial. Bogotá: Universidad del Rosario.

PNUD. (2011). Informe de Desarrollo Humano: Sostenibilidad y Equidad. Nueva York: PNUD.

Ratti, C. \& Townsend, A. (2011). La conexión social. Investigación y Ciencia, (422), 23.

Roca, G. (2012). La sociedad Digital. Conferencia TEDx Galicia. Recuperado de https://www.youtube.com/ watch?v=kMXZbDT5vm0

Salim, I. (2016). How do we fix Civilization? Conferencia TEDx Toronto. Recuperado de https://www.youtube.com/ watch?v=mV0oKVOIGG4

Stiglitz, J. (2017). La globalización del malestar. El País. Recuperado de https:// elpais.com/economia/2017/12/20/ actualidad/1513790732_742783. html

Toro Arango, J. (2000). El ciudadano y su papel en la construcción de lo social. Bogotá: Alcaldía Mayor de Bogotá, Secretaría de Gobierno, Departamento Administrativo de Acción Comunal,

Gloria IsABel Quintero PÉrez 
Veeduría Distrital: Pontificia Universidad Javeriana, Instituto de Estudios Sociales y Culturales-Pensar, CEJA.

Vegara, A. (2009). Territorios Inteligentes. Ambienta, 89, 34-58.

Vegara Gómez, A. \& De la Rivas Sanz, J. L. (2016). Supercities. La inteligencia del territorio. Madrid: Fundación Metrópoli, 390 pp., ISBN 978-84-608-4460-0. 